\title{
Paper mill-derived cancer research: the improbability of prostate cancer in women, and ovarian and breast cancer in men
}

\author{
Jaime A. Teixeira da Silva
}

Independent researcher, Kagawa-ken, Japan

Paper mill-derived research has penetrated biomedical literature, and it is affecting the integrity and reliability of research [1]. The use of paper mills (i.e., pay-to-create services) is an act of misconduct if the use of such services is not declared since it gives the false impression of the authors' effort, input, and originality, when in fact none was involved; moreover authorship is false since the data is created or fabricated by others, and not generated honestly in a laboratory by the authors themselves. Paper mill-derived research has itself become an academic cancer in urgent need of a cure and solutions.

Several sleuths, anonymous and named, continue to examine cancer literature, and their efforts may or may not be related to the cancer reproducibility project by the Center for Open Science. Independent of the source of these discoveries, the discoveries themselves are cause for concern and alarm. In the most recent paper mill exposé, which may or may not be related to other paper mills, three papers are highlighted in this letter: Hu et al. (2018), Pan et al. (2019), and Liu et al. (2020) [2-4]. What is curious about these papers was the discovery of breast cancer in males (Hu et al. [2]), ovarian cancer in males (Liu et al. [4]), and prostate cancer in females (Pan et al. [3]). In all three studies, genders were described as binary, i.e., exclusively biological male and female, and none of the study subjects were indicated as being transgender, which might be associated with altered hormone levels [5] and thus the possibility of confusing genders.

While it is not unusual to discover breast cancer in males, it is a very rare (about $1 \%$ of all cases of breast cancer) phenomenon [6]. Despite this, Hu et al. [2] reported a > 59\% incidence of breast cancer in males (38/64 subjects), apparently detected using a quantitative real-time polymerase chain reaction (qRT-PCR) with a long non-coding RNA (InCRNA), LINC01116. Not only the extremely high value, but also the fact that cases in males were higher than in females (a potentially first extraordinary finding in the cancer literature) suggest that these findings are too good to be true, or, in other words, false.

In Liu et al. [4], ovarian cancer was found in 28 males among 49 subjects, i.e., $>57 \%$ incidence, as apparently detected by LINC00675, while in Pan et al. [3], prostate cancer was found in 27 females among 52 subjects, i.e., an almost 52\% incidence, as apparently detected by microRNA-605-3p, both using qRT-PCR. In these cases, the incidence of ovarian cancer in males and prostate cancer in females should theoretically be $0 \%$, since, evidently, biological males do not have an ovary while biological females do not have a prostate. Would it thus be safe to assume that these findings are either extraordinary, or that they are false? The clue may lie in the fact that Pan et al. (2019) has already been retracted for very opaque reasons.

\section{How to cite:}

Jaime A. Teixeira da Silva. Paper mill-derived cancer research: The improbability of prostate cancer in women, and ovarian and breast cancer in men. NOWOTWORY J Oncol 2021; 71:255-256.

This article is available in open access under Creative Common Attribution-Non-Commercial-No Derivatives 4.0 International (CC BY-NC-ND 4.0) license, allowing to download articles and share them with others as long as they credit the authors and the publisher, but without permission to change them in any way or use them commercially. 
Its retracted status is (unfortunately) not - but should be indicated on its PubMed entry.

This case also draws concern about the journal in which three papers were published - the European Review for Medical and Pharmacological Sciences - which has a 2019 Clarivate Analytics Journal Impact Factor (JIF) of 3.024 and is indexed in the Web of Science and PubMed. The fact that it is an open access journal fortifies the risk of potentially fictitious paper mill-derived cancer research because it is so easy to access, and thus cite. In fact, a Google Scholar search for Hu et al. (2018), Pan et al. (2019), and Liu et al. (2020) [2-4] reveals that they have been cited 44, 4, and 0 times, respectively. In the case of Pan et al. (2019), those citations could be considered to be unfair contributors to the journal's JIF, and the JIF itself would need to be adjusted downwards to account for the retracted paper's citations [7].

This letter provides a bird's-eye view of three papers among dozens or hundreds of papers on cancer with potentially fabricated data and findings, most likely derived from one (and the same) or more paper mills that might have served multiple clients with recycled or fabricated data, including figures, tables, and text, often confusing cancer cell lines within and among papers. Ultimately, readers are left confused, doubt regarding the validity of the findings increases, and mistrust in some of the most basic elements of trust in biomedical and academic publishing, such as the blind claim of the peer review, and the quality aspect of PubMed [8], Clarivate Analytics and the JIF, are now on the increase.

This letter has obvious limitations: it only provides a brief three-paper snapshot of a potentially far-reaching problem regarding the integrity of peer-reviewed and indexed cancer literature. This letter also focuses on one issue almost exclusively, namely the improbability of prostate cancer in women, and ovarian and breast cancer in men, or at least at the levels reported by Hu et al. (2018), Pan et al. (2019), and Liu et al. (2020) [2-4]. There are many other issues in these related papers that need to be explored and discussed.
The criminality of individuals working for and supporting paper mills, and the networks of researchers, editors, journals, and publishers that may be involved, deserves heightened awareness and further investigation.

Confict of interest: none declared

\author{
Jaime A. Teixeira da Silva \\ P. O. Box 7 \\ Ikenobe 3011-2 \\ Kagawa-ken 761-0799, Japan \\ e-mail:jaimetex@yahoo.com
}

Received: 15 May 2021

Accepted: 8 Jun 2021

\section{References}

1. Teixeira da Silva JA. Paper mills and on-demand publishing: Risks to the integrity of journal indexing and metrics. Med J Armed Forces India. 2021; 77(1): 119-120, doi: 10.1016/j.mjafi.2020.08.003, indexed in Pubmed: 33100486.

2. Hu HB, Chen Q, Ding SQ. LncRNA LINC01116 competes with miR-145 for the regulation of ESR1 expression in breast cancer. Eur Rev Med Pharmacol Sci. 2018; 22(7): 1987-1993, doi: 10.26355/eurrev_201804_14726, indexed in Pubmed: 29687853.

3. Pan MZ, Song YL, Gao F. MiR-605-3p inhibits malignant progression of prostate cancer by up-regulating EZH2. Eur Rev Med Pharmacol Sci. 2019;23(20): 8795-8805, doi: 10.26355/eurrev_201910_19274, indexed in Pubmed: 31696466.

4. Liu ZH, Liu WJ, Yu XY, et al. Circ 0005276 aggravates the development of epithelial ovarian cancer by targeting ADAM9. Eur Rev Med Pharmacol Sci. 2020; 24(20): 10375-10382, doi: 10.26355/eurrev_202010_23387, indexed in Pubmed: 33155193.

5. Light AD, Obedin-Maliver J, Sevelius JM, et al. Transgender men who experienced pregnancy after female-to-male gender transitioning. Obstet Gynecol. 2014; 124(6): 1120-1127, doi: 10.1097/ AOG.0000000000000540, indexed in Pubmed: 25415163.

6. Jain S, Gradishar W. Male breast cancer. The Breast. 2018: 974-980.e2, doi: 10.1016/b978-0-323-35955-9.00076-3.

7. Teixeira da Silva JA, Dobránszki J. Citing retracted papers affects education and librarianship, so distorted academic metrics need a correction. J Libr Sch Commun. 2018; 6(1), doi: 10.7710/2162-3309.2258.

8. Teixeira da Silva JA. Is the validity, credibility and reliability of literature indexed in PubMed at risk? Medical J Armed Forces India. 2021, doi: 10.1016/j.mjafi.2021.03.009. 